\title{
Bronquiectasias en el niño
}

\author{
Drs. Iván Cabrera. Víctor Lama. Gonzalo Gómez, Silvia Bark*
}

\begin{abstract}
Fifty cases of bronchiectasis in children admirted ta the Pneurnobos Unit of Luis Ćalvo Mackenna Hospital during 1972 to 1977 ate repurted.

The relatiunship with age, sex. time course before diagnessis, nutritional state, previnus distastes, physieal signs,

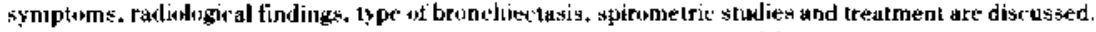

'The obstructive bronchial patholugy was the predominant etiological factor of brunchiectasis in this scudy. Tuberculinite, measles and whouping siugh wer lesw important.

Surgical Irsal meot was rare.
\end{abstract}

Nus pareció de interés hacer una revisión de la bronquiectasia en el niño, presentadas durante los últimos seis años, en la Unidad de Neumotisiologia del Hoepital Luis Calvo Mackenna y comparar nuestros resultados cun trabajos similares hechos en nuestro mismo Servicio, como con otros realizados por otros autores, tanto nacionales como extranjeros. Teníamos la im-

*Servicio de Neumotisiolugia Hosp. Luis Calvo Mackenna presión, a través de nuestra propia experiencia, que esta afección habría variado en cuanto a su etiología, así cumo a la conducta terapéutica frente a ella: concepto que, entre otros, analizamos en el presente trabajo.

Se ha definido a esta afección de diferentes maneras, pero aunando conceptos pensamos que, fundamentalmente, es un proceso que afecta al sistema bronquial, debilitando y destruyendo la estructura 
músculo elástica y el paquete vásculo nervioso de la pared de los hronquios. acarteando la dilatación de ella en forma irreversible, jugando un papel importante en la etiopatogenia de este proceso la infección y la obstruccion bronquial.

No repetiremos aquí su etiopatogenis, ya que eslos conceptos han sido vastamente tratados en publicaciones que hemos hecho anteriormente, asi como por otrus autores.

\section{MATERIAL Y METODO}

Revisamos las bronquiectasias diagnusticadas en la Unidad de Neumotisiologia de nuestro Hospital, entre los años 1972 al 77 inclusive, reuniendo 30 casos. En ellos analizamos: la edad, sexo, tiempo de enfermedad transcurrido antes del diagnóstico, estado nutritivo, enfermedades previas para, en lo posible, precisar su etiklogía, sintumas y signos más relevantes. hallazgos radiolígicus más destacados, tipo de bronquiectasia, tratamiento médico y quirúrgico.

Tratamos en lo posible de hacer un pronóstico aproximado. A todos ellos se les hizo, obviamente. estudio brincográfico y en más del $50 \%$ se les hizo estudio espirométrico.

\section{RESULTADOS}

En la distribución por edad, tuvimos sólo 1 lactante de 1 año 10 meses; 18 preesculares y 31 escolares. 27 casos fueron hombres y 23 mujeres.

En cuanto al tiempo de evolución de su enfermedad antes de establecerse el diagnóstico, encontramos 20 casus $(40 \%)$ con más de 4 años de padecimiento: 20 casus $140 \%$ ) entre 2 y 4 años y 10 casos (20\%) con evolución de menos de 2 años; vale decir, el $80 \%$ tenia más de 2 años de evolución al momento de hacerse el diagnisticot.

\section{Estado nutritivo:}

Un $42 \%$ de ellos eran desnutridos sin precisar el grado de desnutrición.

\section{Enfermedades previas:}

Tratamos de establecer una etiologia clara y encontramos, en algunos casos, que había más de una patologia previa importante, por ejemplo: sarampión y bronconeumonía; coqueluche más brunconeumonia: sarampión y bronquitis recurrente o crónica, etc. En orden decreciente, los resultados fueron los siguientes:
a) Bromulitis obstructica a repeticion yio

crónica: La encontramos en 27 casus (54\%). De ellos 11 casus tenian además el antecedente de sarampión. y 3 rasus, el de cuqueluche.

b) Sarampioin: Lo encontramos en 18 casos. De ellos 7 casos tuvieron además bronconeumonjas repetidas y 1 I casos siguieron con bronquitis a repetición o crónica. En 5 casos presentaron además de sarampión. ambas patologias, es decir, bronconeumonias y branguitis a repeticion.

c) Bronconeumonias a repetición: Las hubo en 18 easos 136\%; 7 de ellas nuvieron además sarampión, y en 3 casos presentaron coqueluche. Hubo 2 casos que presentaron coqueluche. sarampión y bronconeumonías repetidas.

d) Coqueluche: En 6 casos. En 3 de ellos se presentaron además bronconeumınías frecuentes y otros 3 se acompañaron de bronquitis a repetición o crónica.

e) Supuracion pulmonar: Este antecedente se encontró en 6 casos. Dos de ellos fuerun complicaciones de bronconeumonía post sarampión. Uno presentí bronconeumonías repetidas y ninguno se acompaño de broncuitis crónica o a repetición. Esto hace pensar que la brenconeumonia supurada, por sí misma, fue capaz, en algunus casos, de producír bronquiectasias. hecho que ha sido postulado en trabajos anteriores.

f) Tuberculosis pulmonar: Encontramos sujlo 4 casos. Este bajo porcentaje contrasta notoriamente con trabajos anteriores como lo comentaremos posteriormente.

Finalmente, encontramos una miscelania de antecedentes tales como: síndrome de Kartagener, 2 casos: asma bronquial, 3 casos; neumonilis, 2 casos: mucosviscidosis, 1 (aso): arsenicismo crónico, 1 caso: deferto de IGA más escoliosis, 1 caso: quiste pulmonar congénito, 1 caso; sinusitis más bronquitio crónica, l caso. En 1 solo caso no encontramos pato logía previa ni concomitante la que, por ser una bronquitectasia localizada y de tipo quístico, pensamos yue pudiera corresponder a una lesión congénita, sin poder asegurario, ya que existía el antecedente poco claro de un pusible infiltrado TBC tratado en provincia.

\section{SINTOMAS Y SIGNOS}

En forma decreciente, encuntramos lo siguiente:

1. Tos: existió en 48 casos ( $9 \% \%$ ).

2. Estertores húmedus: localizados en $\mathbf{4 3}$ casos $186 \%)$.

3. Expectoración: la presentaron 19 casos (38\%).

4. Dedos hipocráticus: 19 easos (38\%). 
5. Desgarro hemoptoico: lo encontramos en 7 casos (14\%).

6. Hemoptisis: en l solo casu.

7. Disnea: en 6 casos $(12 \%)$.

8. Cianosis: en 4 casos $(8 \%)$.

\section{ESTUDIO RADIOLOGICO}

Con radiografia simple encontramos las siguientes lesiones:

a) Proceso pulmonar crónico con fibrosis: en 34 ca$\operatorname{sos}(68 \%)$.

b) Atelectasia: en 31 casos (62\%).

c) Imágenes areolares: 31 casos (62\%).

Obviamente hubo combinación de lesiones en varios casus, to que explica los purcentajes sobre 100\%. Así encontramos que, en los 32 casos con fibrosis y proceso crónico, presentaron además atelectasias en un alto porcentaje ( 18 casos).

En los 4 casos de TBC pulmonar, 2 presentaban atelectasia y todos ellos una fibrosis crónica difusa.

Broncografía.

Las lesiones fueron bilaterales en 29 casos ( $58 \%$ ) y unilaterales en 2 l casos ( $42 \%$ ).

En las bilaterales las lesiones fueron difusas en 18 casos y localizadas en 11 casos.

Hubo gran predominio de lesiones en el pulmón izquierdo con localización preferente en el L.I.I. y lingula. Le siguen con frecuencia lesiones del L.I.D. acompañados en algunos casos, de bronquiectasias del L.M. Encontramos muy pocas localizaciones en los lóbulos superiores, y casi siempre ellos se comprometieron en bronquiectasias difusas.

Tipode lesion.

El estudio broncográfico reveló lo siguiente:

Bronquiectasias tubulares: 24 casos (48\%).

Bronquiectasias saculares: 21 casos $(42 \%)$.

Bronquiectasias quísticas: 5 casos (10\%).

Tres casos de estas últimas presentaron, además, concomitanternente lesiones tubulares y saculares.

En las bronquiectasias de tipo quistico encontramos como antecedentes, en 2 casos TBC pulmonar; en uno, supuración pulmonar prolongada; en otro, procesu bronquial ubstructivo crónico, y en el tiltimo, sarampión.

\section{Espirometria.}

Se efectuó en 27 casos. Resultó alterada en 22 casos, y normal, en $\mathbf{5}$ casos. La alteración consistió en compromiso obstructivo en 10 casos y restrictivo en 4 casos. En 8 casos hubo compromiso mixto: obstructivo restrictivo.
De los 22 easos con alteraciones espirométricas, 12 fueron operados y de ellos, el $50 \% 16$ casos) en sus cuntroles posteriores siguieron con tos y expecturación. La indicación operatoria fue dada por presentar bronquiectasias localizadas muy avanzadas con supuración crónica.

\section{Tratamiento.}

Obviamente en todos los casos se hizo tratarnient to médico y en 22 de ellos, éste se acompañó de intervención quirúrgica. Consideramos innecesario detallar el tratamiento médico, ya que ha sido descri(1) en forma minuciosa en trabajos anteriores y no difiere de lo publicado por otros autores: sólo nos limitaremos a destacar la enorme importancia que tienen el control periódico, la profilaxis y la kinesioterapia en estos enfermos.

En los 22 casos operados, sólo en 6 se hizo resección tơtal de la lesión, siendo en el resto sólo paliativa, es decir. se eliminó aquella zona más comprometida que constituía una supuración crónica, atelectasia o lesión residual sospechosa de TBC.

Durante los últimos años, la indicación operatoria de estos enfermos ha disminuido, siendo éste actualmente casi de excepción. motivada esta conductu diferente por el cambio en la etiologia en que predominan procesos difusos, frecuentemente obstructivos. El porcentaje, relativamente alto, de nuestra casuistica de 22 enfermos intervenidos, de 50 casus, se explica por la necesidad de indicar resecciones paliativas en casos difusos avanzados con supuraciones crónicas focales atelectasicas o por ser algunos de ellos de provineia, alejados de centros de control.

En los 6 pacientes que se pudo hacer resección total de la lesión, dus de ellos controlados posteriormente, hubo ausencia total de síntonas. Los 4 restantes eran de provincia sin que hayan reingresados posteriormente, hecho que podría suponer una evolución satisfactoria.

De los 16 casos con resección paliativa, 4 eran de provineia, sin tener control posterior de ellos. De los 12 casos controlados, en 9 de ellos persistieron síntomas de tos y expectoración; sin embargo, súlo en 6 de ellos persistieron estertores húmedos localizados; vale decir, en 3 de ellos los sintomas bronquiales pudieron corresponder a bronquitis a repetición u obstructiva asociadas.

\section{Control posterior.}

Fueron controlados posteriurmente 32 casos (64\%). 21 enfermos se controlaron entre 1 a 3 años. (Ya analizamos lo que ocurrió con los operados. I Ana- 
lizaremos ahora los casos que siguieron sólo tratamiento médico, en los cuales se desestimó la operación por ser casos generalmente difusos; con compromiso bilateral impurtante, es decir, los más comprometidos, en que la eliminación de un determinado lóbulo o segmentos, sólo acarrearia un empeoramiento de la función respiratoria por restricción, además del compromiso obstructivo que muchos de ellos tenian. Pero hubo ur pequeño grupo en que sus lesiones fueron poco intensas o de localizaciones altas, en que sabemos que su drenaje es prácticamente espontámeo y permanente, y que no tienen una indicación quirúrgica, salvo excepciones.

En 28 casus de este grupo, 11 siguieron con síntomas y signes atribuibles a su bronquiectasia, 6 de ellos presentaron disnea, 7 con dedos hipocráticos. 2 presentaron cianosis, es decir, correspondian a enfermos de evoluciön prolongada con lesiones avanzadas y difusas. Los 17 restantes se consideraron de evolución satisfactoria. con sintomatologia ocasional, muchas veces en relación a cuadros respiratorios agudos intercurrentes. En ambos grupos hubo algunos con asma y cuya evolución estuvo, en gran parte, determinada por esta enfermedad. Pensamos que el control periódico, con un tratamiento médico bien llevado, más kinesioterapia, es suficiente para mantener a estos enfermos libres de infección y en buenas condiciones generales, siempre que su diagnóstico no sea tardío, con lesiones muy avanzadas.

\section{COMENTARIO}

Todos los autores están acordes que en la etiopatogenia de la bronquiectasia, juegan un papel preponderante la infección y la obstrucción bronquial; y los procesos de alteración de la mecánica y función respiratoria que derivan de estos dos factores básicos. Una vez más destacamos la gran frecuencia del hallazgo de atelectasia, ya sea lobular o segmentaria. La encontramos en un $62 \%$ en la radiografía, acompañando o siguiendo a procesos con patología obstructiva: bronconeumonías o neumonías a repetición, TBC, etc. Fue frecuente el hallazgo de bronquiectasia en los lóbulos inferiores, especialmente en L.I.l., que sigue a una atelectasia crónica de ellos, por lo que consideramos que es un elemento patogénico importante y frecuente en la producción de esta enfermedad.

El estudio nutritivo es considerado factor importante en esta afección. Creemos que la desnutrición condiciona una evolución más desfavorable, ya que por estar las defensas bajas en estos niños, las infecciones respiratorias intercurrentes son más frecuen- tes y de cursu habitualmente más grave y prolongado. Un $42 \%$ de nuestros casos eran desnutridos, sin entrar a precisar su grado de desnutrición.

En cuanto a la etiología nes llamé la atención el bajo porcentaje del antecedente de tuberculosis como factor etiológico. Así, en un trabajo realizado hace algunos años en nuestro mismo Servicio, esta etiología tuvo una incidencia de un $70.6 \%$ comparado con sólo un $10 \%$ en nuestra casuistica actual. Esto es explicable por el mejor control y tratarniento de la TBC. durante los últimos años que ha becho bajar substancialmente la incidencia de ella, especialmente en el niño y a que el material del anterior trabajo, hasta cierto punto fue seleccionado entre enfermos con frecuente patologia TBC. También, enfermedades comı el sarampión y coqueluche, aparecen con bajos purcentajes como patología previa pura, motivado a nuestro entender, por las actuales medidas preventivas (vacunación); pero aún así, en aigunos casoss en que alguna de ellas estuvo presente, jugó un rol importante tanto por sí misma como cuando fue seguida de bronconeumonía supurada u a repetición.

Nuestra casuística reveló gran preponderancia como factor etiológico de la bronquiectasia, los proresos bronquiales obstructivos reiterados o crónicos y neumopatías agudas a repetición $y$ muchas veces presentandose juntos en un mismo enfermo.

Nuevamente hemos encontrado, como otros autores, que la tríada sintomática más constante está constituida por tos, expectoración y el hallazgo de estertores húmedos localizados y persistentes. Otro signo que debe buscarse siempre, ya que acompaña con frecuencia a estos enfermos, ya sea por la enfermedad causal o la bronquiectasia misma de evolución prolongada ${ }_{+}$es el hipocratismo el que estuvo presente en un porcentaje importante en nuestros enfermos $138 \%)$.

La hemoptisis, signo siempre destacado en las bronquiectasias, estuvo presente en muy escaso porcentaje y fue reemplazado por la presencia más bien de desgarro hemoptoico. Fue franca en un sólo caso.

No encontramos ningún caso con base clínica y radiológica suficiente como para catalogarla de bronquiectasia congénita.

En cuanto al tratamiento operatorio de esta afección, ha sido notorio durante los últimos años, su menor indicación, quedando reducida sólo a aquellos casos bien localizados, generalmente secundarios, a una atelectasia de lúbulos inferiores o secundaria a una TBC localizada, o también en procesos localizados muy avanzados que constituyen una supuración crónica, aun cuando sean parte de un proceso más 
difuso. Pensamos que el predominio de la patologia obstruc-liva y rónica como etiología más frecuente $y$, por lo lanto, la presencia de procesos difusos y bilaterales, ha hecho menos frecuente el tratamiento quirúrgico, el cual súlu causaria mayor alteración de la función respiratoria por restricción, la que se sumaria a la obstruccioin preexistente en estos enfermos.

Todo eslo hace que tome la mayor importancia el tratamiento médico, haciendo hincapiê en algunos puntus fundamentales, destacando la profilaxis de la infección. control periưtico y permanente de eslus enfermos, un buen tratamiento y profilaxis de las afecriones causales. buen manejo de la patologa obs. tructiva y destacamos. en forma muy especial. la kinesioterapia respiratoria. *.

\section{RESUMEN}

Se revisan 50 casos de bronquiectasias en niños ingresados a la Unidad de Neumotisiologia del Hospital Luis Calvo Mackenna, entre los años 1972 a 1977. En ellos se analizan; edarl, sexa. tiempo de enfermedad transcurrido antes del diagnóstico, estado nutritivo, enfermedades previas para tratar de precisar su etiologia: síntumas y signos más relevantes. hallazgus radiológicos más destacados, tipo de bronquiectasia y tratamiento. En todus se hizo estudio radiológico y en más del $50 \%$ se le hizo estudio espirométrico.
Se destaca en esta revision el predominio de la patolugía bronquial obstructiva como causa de bronquiectasia, condicionando un compromiso bronquial más difuso; así como la gran disminución de la etiologia tuberculosa, del sarampión y de la coqueluche.

El tratamiento quirúrgico se ha hecho menos frecuente.

\section{REFERENCIAS}

${ }^{1}$ Bual, L. y cof.: "Bronquiectasias en al Niño, Estudio clínicaty bruncogáfice en 60 Lasus". Rev. (.hil. Pedtatr. 45: 127, 1974.

${ }^{2}$ Contun, C.: "Cunsiderations sur le traitenent des bronchipvtasies bilacerales chez l'enfant pur Restrtion pilınonaire asgocize bilate-

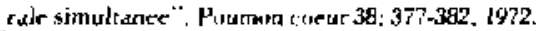

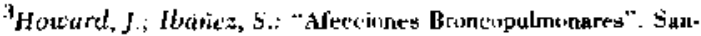
(iago), Editurial de la Universidad (:atiolica de C'hile. 1965.

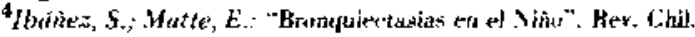
Pedia1r. 32: 551. 1961

5Menezhello. J.: "'Tralado de Prdiatria". Intermedica, T-2: 78-83. ]Y7.2.

${ }^{6}$ hobbins, S.+ Tratado de patulogía con aplicsciún clínica. Mexicur. Editurial Interamericana \$. A., 1\%33.

'Kendik, E.: Puknimary Disorders. Philadelphia. Editurial W. B. Saunders Cumpany, 1972.

Afeld, E,: Bromohiectasis. Third report on a follow up study of medical and surgiral cases from childhund. Are. Dig. Chil. 44: 55l. 1969.

${ }^{9}$ Carressev, E.: Branyuiectagias. Bul. Hosp. San Juan de Dios 11 : 173. 1964.

${ }^{10}$ Burdacth, R.: Erjolengia de bronquiectusias en Hospital M. Arriarin. 141 casos (1946-1963). Conjunicucisin persinal. Nap publit'ada.

IIfar, H.: Tratamiento de Bronquiectasias. Dish. Med. Werh, 93 $138,156 \mathrm{~d}$. 